\title{
Comunicación
}

\section{CONTRIBUCIÓN AL CONOCIMIENTO DE LA BIOLOGÍA DE LA CORVINA Plagioscion squamosissimus (PERCIFORMES: SCIAENIDAE) DE UCAYALI}

\author{
Contribution to Knowledge of the Biology of Corvine Plagioscion \\ squamosissimus (Perciformes: SCIAENidae) in UCAYAli
}

José C. Riofrío Q. ${ }^{1}$

\section{Resumen}

Se analizaron 321 ejemplares de la corvina Plagioscion squamosissimus entre julio de 2003 a junio de 2004 con la finalidad de obtener la relación longitud - peso, proporción por sexos, época de desove, talla media de madurez (longitud total), y composición de su item alimenticio. La relación longitud - peso no mostró diferencias significativas entre sexos, proponiéndose la ecuación conjunta $\mathrm{PT}=0.0057708 \mathrm{LT}^{3.21}$ para la especie. La proporción sexual se ajustó a la proporción 1:1. La talla media de madurez en machos fue de $29.6 \mathrm{~cm}$ y para hembras de $31.2 \mathrm{~cm}$. Las variaciones del índice gónadosomático (IGS) evidenciaron que la especie se reproduce durante todo el año, aunque con mayor frecuencia entre setiembre y octubre (época de vaciante e inicios de creciente) y en menor cuantía entre febrero y marzo (mediados a fines de creciente). La dieta estuvo conformada fundamentalmente por peces.

Palabras clave: Amazonía peruana, Ucayali, corvina, Plagioscion squamosissimus, manejo pesquero

\section{AbSTRaCt}

In the study, 321 individuals of Plagioscion squamossissimus were analyzed from July 2003 to June 2004 to obtain the length - weight relation, sex ratio, egg-laying seasonality, mature size, and composition of their diet. The length - weight relation did not show significant differences between sexes, and therefore the combined equation PT $=0.0057708 \mathrm{LT}^{3.21}$ is proposed for the species. The sex ratio was close to $1: 1$. The mature size was $29.6 \mathrm{~cm}$ in males and $31.2 \mathrm{~cm}$ in females. Variations in the gonad-somatic index (IGS) showed that this species reproduce throughout the year, but more frequently between

1 Facultad de Ciencias Biológicas, Universidad Nacional Mayor de San Marcos, Lima. E-mail: jriofrioq@unmsm.edu.pe,jriofrioq@yahoo.com 
September and October (end of dry season) and between February and March (mid and end of rainy season). The diet of the species was mainly composed by fishes.

Key words : Peruvian Amazon, Ucayali, corvina, Plagioscion squamossissimus, fisheries management

Plagioscion es un género de peces de escamas, de hábitos alimenticios piscívoro, de la familia Sciaenidae. En la cuenca amazónica peruana se reporta $P$. squamosissimus y P. auratus (Ortega y Vari, 1986); ambas, especies de corvina de valor comercial para consumo humano directo. Información de desembarques de estas especies en el puerto de Pucallpa, en estado fresco y refrigerado entre 1994 y 2002 indican que la mayor colección ocurrió en el año 1995, donde su aporte constituyó el 5.4\% de los desembarques totales, y a partir de allí descendió el volumen de corvina capturada ( $0.1 \%$ del desembarque total en el 2001$)$. Asimismo, el mayor volumen de captura llega al puerto de Ucayali en estado refrigerado.

El descenso en los volúmenes de captura es un claro indicio de la sobreexplotación del recurso. En la actualidad, la corvina $P$. squamosissimus no se explota en la piscicultura; sin embargo, dada la excelente calidad de su carne se espera que en un futuro cercano se incluya entre las especies cultivadas. En la Amazonía Central se le considera como uno de los mejores pescados (Loubens y Aquim, 1986) por ser especie magra y con tenor de proteína de $19 \%$.

A pesar de su importancia comercial y potencial piscícola, se han realizado pocos estudios sobre su biología en la cuenca del Ucayali. El presente estudio se llevó a cabo con la finalidad de incrementar los conocimientos sobre las especies amazónicas en el Perú y contribuir al manejo de la pesquería de esta especie.

Se analizaron 321 ejemplares de $P$. squamosissimus "corvina", provenientes de los mercados de Pucallpa (Mercado
Bellavista, Mercado N. ${ }^{\circ}$ 2, Puerto La Hoyada y Puerto de Yarinacocha). Los especímenes se evaluaron en el Laboratorio de Pesquería del Centro de Investigación IVITA - Pucallpa, de la Universidad Nacional Mayor de San Marcos. El periodo de estudio abarcó entre julio 2003 y junio 2004.

Se determinó la longitud total (LT) y peso total (PT) de los ejemplares usando un ictiómetro de $0.5 \mathrm{~cm}$ y una balanza digital con $0.1 \mathrm{~g}$ de precisión para obtener la relación longitud - peso por sexo, empleando la ecuación alométrica $\mathrm{P}_{\mathrm{t}}=\mathrm{a} \mathrm{Lt}{ }^{\mathrm{b}}$ (Ricker, 1975). Esta relación se analizó mediante regresión lineal de los logaritmos de la longitud total y peso total, para sexos por separado y agrupados (Le Cren, 1951; Sparre y Venema, 1992). Asimismo, se analizó el contenido estomacal identificando los ítemes hasta el taxón más bajo posible.

Los aspectos reproductivos consideraron el sexo, estadio de madurez y peso de las gónadas (PG), y se analizaron con la prueba del Chi cuadrado. La variación del índice gónado somático (IGS) se calculó mediante la fórmula: Peso de la gónada del pez $(\mathrm{g}) \mathrm{x}$ 100 / peso corporal total (g), que permite determinar la época de reproducción al observarse la caída de los valores del IGS, mientras los estadios de madurez asociados a las tallas permiten determinar la talla media de desove. Se utilizó la escala de Holden y Raitt (1974) empleada para peces tropicales de desove parcelado: Estadio I: inmaduros o vírgenes; Estadio II: inicio de la maduración de vírgenes y de desovados en recuperación; Estadio III: en maduración o desarrollo y desarrollado; Estadio IV: grávida; Estadio V; desove; Estadio VI: desovado (desarrollo asincrónico de los ovocitos). 


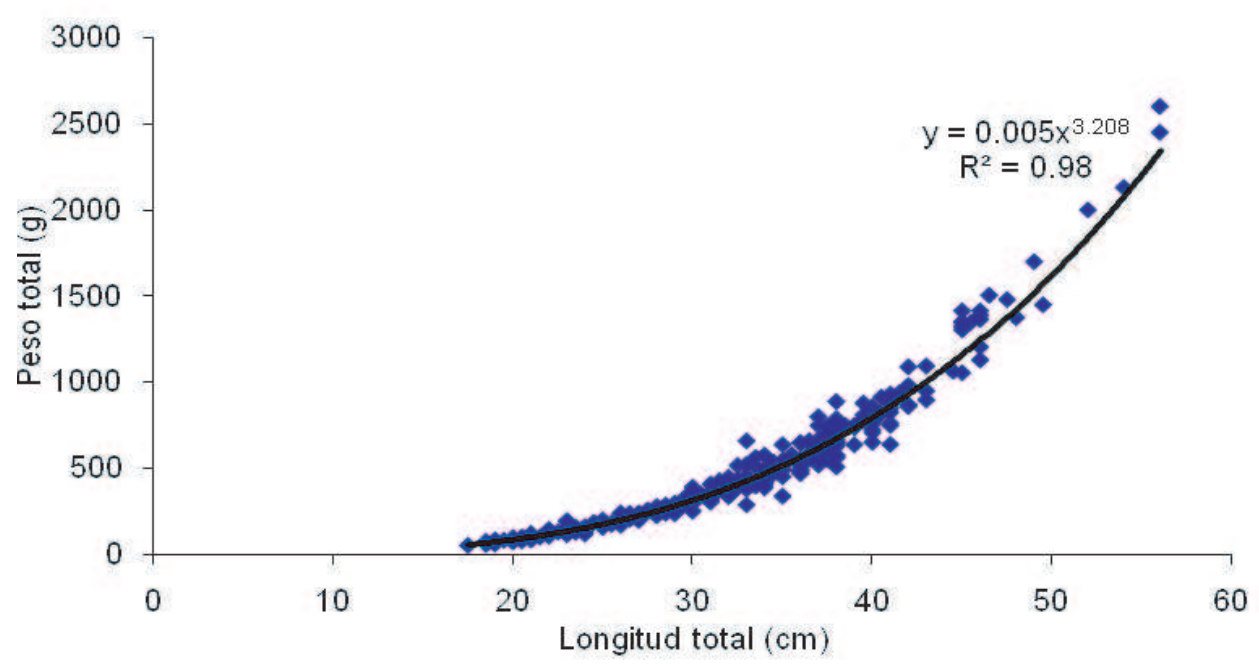

Figura 1. Relación Longitud-Peso de la corvina Plagioscion squamosissimus para pbolaciones combinadas de machos y hembras ( $\mathrm{n}=321$, Ucayali, Julio 2003 - junio 2004)

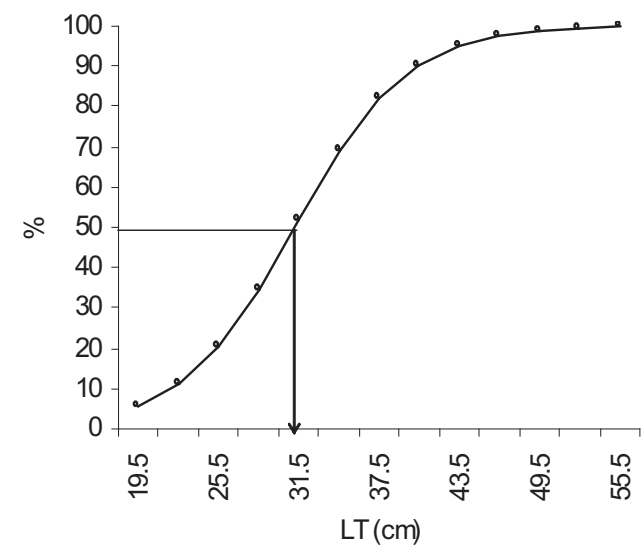

Machos

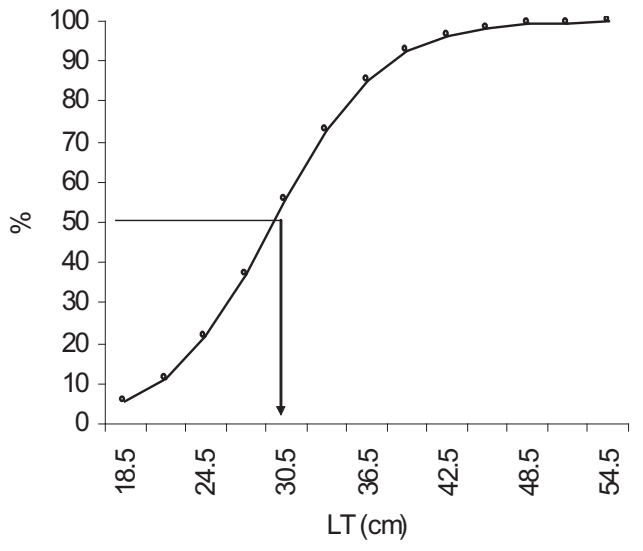

Hembras

Figura 2. Talla media de madurez de la corvina Plagioscion squamosissimus de Ucayali, según sexo (Ucayali, julio 2003 - junio 2004)

Los diagramas de dispersión de los datos mostraron una relación exponencial por lo que se empleó la ecuación $\mathrm{PT}=\mathrm{a} \mathrm{LT}^{\mathrm{b}}$ para correlacionar las dos variables y obtener los valores de a y b. El análisis de varianza demostró que no existen diferencias significati- vas entre las regresiones de sexos independientes y la regresión con sexos combinados, proponiéndose utilizar la ecuación para sexos combinados como la ecuación para la relación longitud - peso para la especie: $\mathrm{PT}=$ 0.0057708 LT $^{3.21}$ (Fig. 1). 


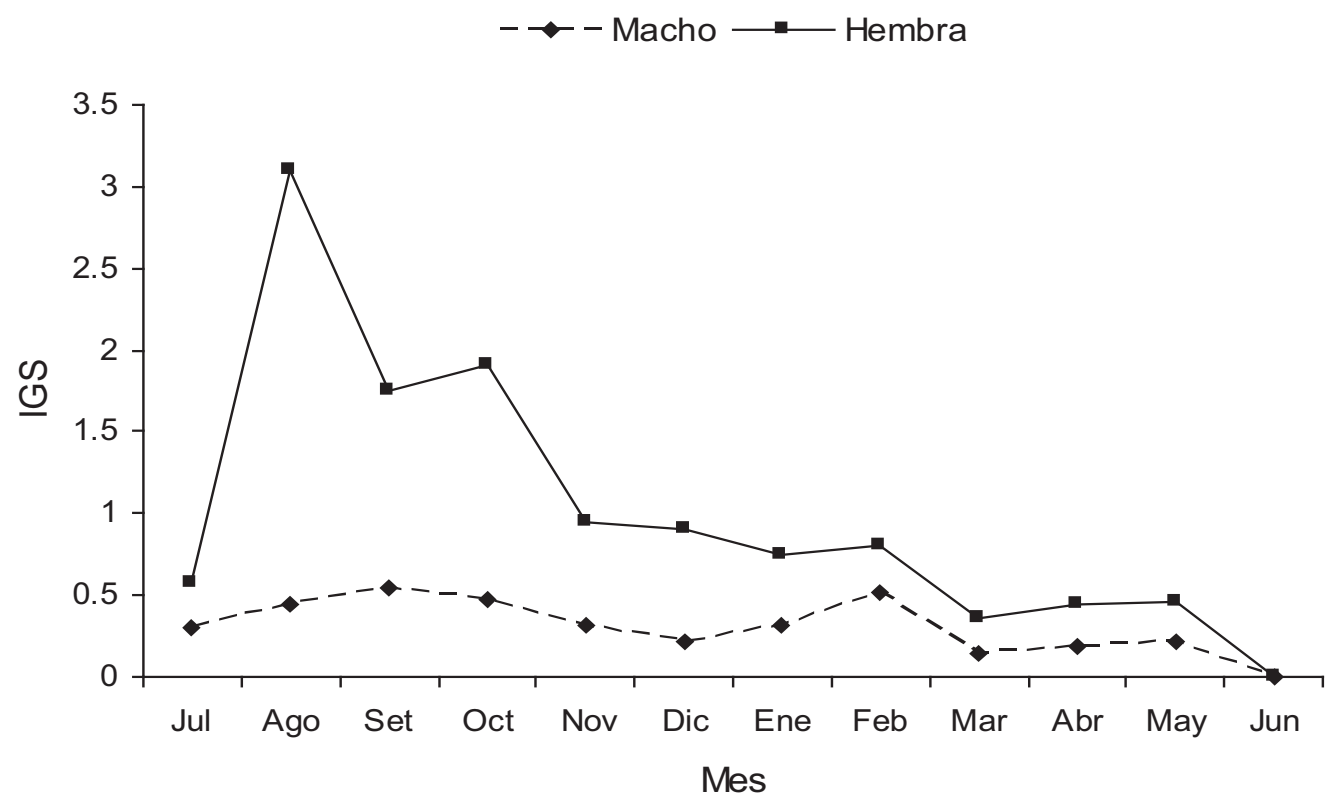

Figura 3. Variación mensual del índice gonadosomático de la corvina Plagioscion squamosissimus en la región de Ucayali, según sexo (julio 2003 - junio 2004)

Los datos trimestrales se analizaron mediante la prueba de Chi cuadrado para observar diferencias entre la proporción de machos y hembras, la cual es asumida de ser 1:1, no hallándose diferencias significativas $($ machos $=156$, hembras $=165)$.

Las medidas de la talla (LT) de la especie se agruparon en frecuencia de longitudes para 14 grupos de LT, considerando los estadios II a VI para hembras y machos. La talla media de madurez fue de $31.2 \mathrm{~cm}$ LT para hembras y $29.6 \mathrm{~cm} \mathrm{LT}$ para machos (Fig. 2). Estos resultados indican que las hembras alcanzan tallas de madurez ligeramente superiores a los machos, resultado que coincide con lo obtenido por Reyes et al. (2003) en Bajo Orinoquia, Colombia, aunque ellos encontraron diferencias de $10 \mathrm{~cm}$.

La época de desove, en el caso de hembras, muestra un pico importante en el mes de agosto, según lo demuestra la caída del índice gónado somático (Fig. 2), y de allí la frecuencia de hembras en desove va disminuyendo hasta llegar a su punto más bajo en junio. En el caso de machos, el Índice Gonadosomático es más parejo a lo largo del año aunque se observa un incremento hacia el mes de setiembre, y otra elevación en febrero (Fig. 3). Este comportamiento de los valores del IGS indica que la corvina es de desove parcelado, pudiéndose asumir que la época principal sería durante los meses de setiembre-octubre y otra en febrero-marzo, que son épocas de finales de vaciante e inicios de creciente en el primer caso, y mediados a fines de creciente en el segundo.

Goulding (1980) señala que la reproducción de la corvina ocurre durante todo el año, pero Nico y Thaphorn, 1984 y Novoa, 1982 (citados por Reyes et al., 2003) indican que si bien se reproduce a lo largo del año, presenta un pico en octubre (final de las lluvias) $y$ otro en julio (inicio de las lluvias), coincidiendo parcialmente con los resultados del presente estudio. Asimismo, se indica que esta especie se caracteriza por puestas secuenciales, lo que ofrece posibilidades de resistir a una eventual sobre explotación, en parte debido a que no muestran cuidado 
parental y los juveniles pueden colonizar nuevos hábitat rápidamente, aún en condiciones ambientales desfavorables y bajo alta presión predatoria (Loubens y Aquim, 1986; Rufino e Isaac, 1995).

De 249 ejemplares analizados, 201 tenían el estómago vacío o el contenido estomacal se hallaba en avanzado estado de digestión y algunos otros presentaban restos semi digeridos. Es así que solo se contó con 35 ejemplares en cuyos estómagos se pudo identificar los siguientes ítems alimentarios: Psectrogaster rutiloides (11), Characiforme (5), Gymnotiforme (4), Macrobrachium sp. (2), Potamorhna altamazonica (2), Callichthyidae, Characidae, Curimatella sp., Curimatidae, Hemiodus amazona, Moenkhausia sp., Opsodoras sp., Pellona castelnaeana, Doradidae, Siluriformes, Triportheus angulatus. Asimismo, en otros dos casos se observó restos vegetales.

La dieta de $P$. squamosissimus se basa principalmente en peces, destacando la especie Psectrogaster rutiloides "chiochio" como un componente importante. La presencia de una gran variedad de especies en el contenido estomacal demuestra una composición heterogénea coincidiendo con otros estudios (Reyes et al., 2003). Esto sugiere que la especie explora distintos hábitat en busca de su alimento, aprovechando la oferta del sistema, siendo los restos vegetales un ítem cuya presencia se puede deber a la acción predatoria de la corvina en un ambiente con presencia de macrófitas. Asimismo, Pimelodus y los Curimátidos se hallan asociados a las riberas entre gramíneas y ciperáceas, principalmente Paspalum, Eleocharis y Dichromena, lo que indica que el consumo de plantas sería un ítem incidental (Machado-Allison, 1993).

Si bien es cierto, los resultados muestran a una especie predominantemente piscívora, diversos estudios indican que los ambientes de agua dulce ofrecen pocas oportunidades para la especialización de los peces y, como consecuencia, muchas especies presentan amplia flexibilidad en relación a los diferentes tipos de hábitat y hábitos, compartiendo con otras especies los recursos disponibles en el ambiente (Larkin, 1956, Bezerra et al., 2002, Pereira et al., 2004). Esta capacidad adaptativa de la corvina a nuevos ambientes debe de analizarse para eventuales cultivos extensivos o semi intensivos, profundizando los estudios sobre las posibles interacciones con la ictiofauna local.

\section{Agradecimientos}

El autor expresa su agradecimiento al Instituto Tecnológico Pesquero del Perú (ITP) por el apoyo económico para la ejecución del estudio, así como al Tco. Víctor Muñoz Pezo por su apoyo en los muestreos biológicos.

\section{Literatura Citada}

1. Bezerra GH, Dias F, Gurgel de L. 2002. Dieta de sete espécies de peixes do semi-árido do Rio Grande do Norte, Brasil. Rev Ictiol 10(1/2): 7-16 [Internet], [20 agosto 2006]. Disponible en : http:// vet.unne.edu.ar/inicne/revictiol/ 2\%20BEZERRA/bezerra.htm

2. Goulding M. 1980. The fishes and the forest. Explorations in Amazonian natural history. Los Angeles, USA: University of California Press. 280 p.

3. Holden MJ, Raitt DFS. 1974. Manual of fisheries science. Part 2. Methods of resource investigation and their application. FAO Fish Tech. Paper No. 115 (Rev. 1). Rome: FAO. 214 p.

4. Larkin PA. 1956. Interspecific competition and population control in freshwater fish. J Fish Res Bd Can 22: 1357-1377.

5. Le Cren E. 1951. The length-weight relation and seasonal cycle in gonad weight and condition in the perch, Perca fluviatilis. J Anim Ecol 20: 201-219.

6. Loubens G, Aquim JL. 1986. Sexualidad y Reproducción de los principales peces de la Cuenca del Río Mamoré, Beni, Boli- 
via. Informe Científico N. ${ }^{\circ}$ 5. Convenio ORSTOM-UTB-CORDEBENI. $45 \mathrm{p}$.

7. Machado-Allison A. 1993. Los peces de los llanos de Venezuela: Un ensayo sobre su historia natural. Caracas: Ed Litopar. $137 \mathrm{p}$.

8. Ortega H, Vari RP. 1986. Annotated checklist of the freshwater fishes of Peru. Smithsonian Contributions to Zoology 437: 1-25.

9. Pereira CCGF, Smith WS, Espíndola ELG. 2004. Hábitos alimenticios de nueve especies de peces del embalse de Três Irmãos, São Paulo, Brasil. Universidad y Ciencia. Número Especial I: 33-38. [Internet], [20 agosto 2006]. Disponible en: www.ujat.mx/publicaciones/uciencia

10. Reyes H, John J, Ramírez HG 2003. Aspectos biologicos de sapuara Semaprochilodus laticepas (Steinda-chner, 1879), curvinata Plagioscium squamosissimus
(Heckel, 1840) (Pisces - Characiformes) y sierra copora Oxidoras niger (Valenciennes, 1833) (Pisces - Siluriformes) procedentes de la Baja Orinoquia. [Internet], [23 agosto 2006]. Disponible en: http://www.iiap.org.pe/publicaciones/ CDs/MEMORIAS_VALIDAS/pdfs/ Reyes.pdf

11. Ricker WE. 1975. Computation and interpretation of biological statistics of fish populations. Bull Fish Rca Boani Can 191: 1-382.

12. Ruffino ML, Isaac VJ. 1995. Life cycle and biological parameters of several Brazilian Amazon fish species. Naga ICLARM Q 18(4): 41-45.

13. Sparre P, Venema SC. 1992. Introduction to tropical fish stock assessment. Part 1. Manual. FAO Fisheries Technical Paper No 306.1 (Rev. 1). Rome: FAO. 376 p. 